\title{
Efecto de un programa de bipedestación con estabilizador distal sobre el equilibrio estático y dinámico en bípedo en un caso de parálisis cerebral. Pereira, 2019.
}

\author{
Dilyan Nathaly Arcila Agudelo'; Karol Tatiana Tobón Valencia²;
}

Claudia Jimena López ${ }^{3}$; Jhonatan González Santamaría ${ }^{4}$

RESUMEN Introducción: la parálisis cerebral (PC) es un trastorno del desarrollo de la postura y el movimiento, que presenta como principal característica la actividad postural anómala, lo cual es sinónimo de pobre desempeño en el equilibrio estático y dinámico. Por tanto, los niños con secuelas de PC presentan menor funcionalidad en bípedo, dependencia, riesgo de pérdida de balance y caídas.

Métodos: el estudio es un diseño experimental de caso clínico intrasujeto A-B (muestra $\mathrm{n}=1$ ) longitudinal prospectivo, en el que la variable independiente es la aplicación de un programa de bipedestación con estabilización distal y las variables dependientes son las relacionadas con el equilibrio estático y dinámico en bípedo.

Resultados: dentro de los resultados más significativos fueron los evaluados con la escala de Berg Pediátrica, donde se encontró una mejoría en 9 categorías. Por tal motivo, el puntaje total post-intervención superó en 12 puntos a la inicial, lo cual indicó una mejoría tanto en el equilibrio estático como dinámico; además, otras variables evaluadas también mostraron progresión, y las restantes mantuvieron el puntaje inicial.

Parálisis Cerebral;

Equilibrio;

Estabilizador Distal

Estudiante programa de Fisioterapia, Fundación Universitaria de Área Andina.

2 Estudiante programa de Fisioterapia Fundación Universitaria de Área Andina.

3 Magister en Neurorehabilitación, Docente programa de Fisioterapia Fundación Universitaria de Área Andina. Clopez71@areandina.edu.co

4 Doctor en Ciencias de la Educación, Docente programa de Fisioterapia Fundación Universitaria de Área Andina. Jsantamaria13@areandina.edu.co 


\title{
Effect of a distal stabilizer sit-up program on static and dynamic equilibrium in bipeds in a case of cerebral palsy. Pereira, 2019.
}

\begin{abstract}
Introduction: cerebral palsy (CP) is a disorder of the development of posture and movement, which has as its main characteristic abnormal postural activity, which is synonymous with poor performance in static and dynamic equilibrium. Therefore, children with PC sequelae have lower bipedal functionality, dependence, risk of loss of balance and falls.

Methods: the study is an experimental design of a prospective intrasubject $A B$ clinical case (sample $n=1$ ), in which the independent variable is the application of a distal stabilization standing program and the dependent variables are those related to static equilibrium. and dynamic in biped.

Results: among the most significant results were those evaluated with the Pediatric Berg scale where an improvement was found in 9 categories. For this reason, the total post-intervention score exceeded the initial one by 12 points, which indicated an improvement in both static and dynamic equilibrium; In addition, other variables evaluated also showed progression and the remaining ones maintained the initial score.
\end{abstract}

Cerebral Palsy;

KEYWORDS Balance;

Mini Standing. 


\section{INTRODUCCIÓN}

El término de PC no es un diagnóstico específico, es un amplio abanico que incluye múltiples formas patológicas. Se define como un trastorno del desarrollo de la postura y el movimiento (1), el cual presenta como principales características los patrones anómalos de movimiento y postura, la deficiencia de las reacciones posturales, la mala coordinación y regulación del tono muscular. Este cuadro clínico la convierte en la principal causa de discapacidad infantil (2). Debido a estas deficiencias funcionales, los niños con secuelas de PC presentan limitaciones para su desempeño en bípedo, dependencia, riesgo de pérdida de balance y caídas, generando diferentes tipos de restricciones, desde la movilidad independiente hasta la participación social, partiendo así, de una condición de deficiencia hacia una discapacidad.

Para favorecer los patrones funcionales y el mejor desempeño en el equilibrio y el control postural, se utilizan diferentes estrategias terapéuticas; algunas fundamentadas en las técnicas convencionales y otras fundamentadas desde los nuevos modelos del control motor. Entre estas últimas, unas son costosas y/o implican el uso de tecnología. Pero también, dentro de este grupo aparece en los últimos años el estabilizador distal o "mini standing" (según su nombre en inglés, presentado así por los fisioterapeutas españoles Lourdes y Fagoaga). Esta estrategias tipo órtesis, que facilita la bipedestación, el entrenamiento de las reacciones posturales, el reclutamiento de los músculos antigravitatorios y el reconocimiento de los ajustes posturales necesarias para un buen desempeño del control postural(3)(4). Teniendo en cuenta estos beneficios que según la bibliografía esta estrategia terapéutica presenta a nivel de las funciones relacionadas con el control postural, su uso influenciaría en el desempeño de los individuos en las actividad y su participación, favoreciendo el desarrollo general del niño.

Debido a que hay escasa evidencia sobre esta estrategia en Colombia existe una gran cantidad de dificultades económicas para acceder a tecnologías en los procesos de rehabilitación, se considera preciso indagar sobre el impacto de un programa de bipedestación en el equilibrio estático y dinámico, implementando un estabilizador distal o mini standing, en un niño con diagnóstico de PC en el departamento de Risaralda, 2019.

\section{METODOLOGÍA}

El estudio es un diseño experimental de caso clínico intrasujeto A-B (muestra $n=1$ ) longitudinal prospectivo, en el que la variable independiente es la aplicación de un programa de bipedestación con estabilización distal y las variables dependientes son las relacionadas con el equilibrio estático y dinámico en bípedo.

El sujeto de estudio es una niña con diagnóstico médico de parálisis cerebral infantil tipo diparesia espástica de 8 años de edad, quien presenta discapacidad motora, ubicada en el nivel III en el sistema de clasificación de la función motora gruesa para parálisis cerebral infantil (Gross Motor Function Classification System GMF$\mathrm{CS}$ ), lo cual significa que está en proceso de adquisición de su patrón de marcha, pero requiere apoyo, quien presenta deficiencia en el equilibrio estático y dinámico, 
con buen desempeño de las funciones mentales superiores, capacidad de entender, comunicación verbal y seguimiento de instrucciones. Además, se contó con la aceptación de consentimiento informado por parte de los padres.

Para la evaluación y calificación del desempeño en el equilibrio estático y dinámico, se realizó evaluación previa y posterior a la intervención, utilizando los siguientes test: Test de caminata de 6 minutos, el cual cuantifica la movilidad funcional basada en la distancia recorrida en metros en seis minutos. Durante la prueba, se cuantifican las siguientes variables fisiológicas: frecuencia cardiaca, frecuencia respiratoria, saturación de oxígeno, la presión arterial sistólica y la presión arterial diastólica. También se percibe el esfuerzo del niño utilizando la escala de Borg pediátrica (6).

Test de Timed up-and-go (TUG), el cual cuantifica la movilidad funcional basada en el tiempo (en segundos) que requiere un individuo para llevar a cabo la tarea de levantarse de una silla sin apoyabrazos, caminar tres metros, darse la vuelta, volver hacia la silla y sentarse de nuevo (7).

Test del alcance funcional modificado, adaptación realizada de la prueba en adultos, explora el control del equilibrio dinámico, al medir el desplazamiento anterior capaz de realizar sin modificar la base de sustentación (7).

Escala de equilibrio de Berg, la cual evalúa el equilibrio funcional, mediante exploración de actividades similares a las de la vida diaria. Los artículos se califican en una escala de cinco puntos $(0,1,2,3$ o 4). El cero denota una incapacidad para realizar la actividad sin ayuda y cuatro que expresa la capacidad de realizar la tarea con independencia (8).

Además, para clasificar el desempeño global en la función motora gruesa, se utilizó el Sistema de Clasificación de la Función Motora Gruesa [Gross Motor Function Classification System (GMFCS)]. Este sistema se basa en 5 niveles de clasificación, los cuales hacen énfasis en las capacidades funcionales; el nivel I representa la función motora gruesa más alta, mientras que el nivel $\mathrm{V}$ representa la más baja (9).

El proceso de recolección de información, se desarrolló en cinco etapas. En la primera se realizó la selección de la paciente y la firma del consentimiento informado por parte de los padres de la menor. En la segunda se llevó a cabo la evaluación fisioterapéutica previa a la intervención, donde además de evaluar el equilibrio estático y dinámico con las estrategias ya presentadas, se evaluaron las condiciones neuromusculares: el tono se cuantificó con la escala de Tardieu, la fuerza muscular fue cuantificada con la MRC (Medical Research Counsil) y los rangos de movimiento y flexibilidad muscular fueron cuantificados con mediciones de goniometría.

Posterior a esto, se llevó a cabo una tercera fase en la cual se diseñó el estabilizador distal o mini standing según las necesidades de la paciente. La cuarta fase consistió en la implementación del programa de intervención con el uso del estabilizador distal o mini standing, el cual fue diseñado según las necesidades identificadas en la examinación. Este programa tuvo una duración de 6 semanas, con una intensidad de 5 veces a la semana, durante 60 minutos, usando el estabilizador distal o mini standing. El tiempo de trabajo estaba distribuido de la siguiente manera: 30 minutos con 
acompañamiento desde Fisioterapia, desarrollando actividades de alcances sobre y fuera de los límites de estabilidad en todas las direcciones, promoviendo la activación de las reacciones posturales y los músculos anti gravitatorios, transiciones de sedente a bípedo y viceversa, y 30 minutos en actividades de la vida diaria supervisadas por el cuidador, para lo cual fueron capacitados previamente. En la última etapa se realizó la evaluación pos intervención, siguiendo las mismas estrategias desarrolladas en la evaluación inicial.

\section{RESULTADOS}

Respecto a los resultados obtenidos pre y post-intervención en las diferentes variables, se pudo observar que para la evaluación del tono muscular se mantuvo el puntaje de la espasticidad según escala de Tardieu, el cual fue de 2 para los aductores y flexores de rodilla y plantiflexores bilateralmente; en cuanto al seguimiento de los ángulos de reacción muscular, se evidenció un amento en el ángulo de reacción muscular (R1) al estiramiento rápido de los flexores de rodilla bilateralmente, con una ganancia de $10^{\circ}$, lo cual significa una mayor amplitud del rango de movimiento permitido por el tono dinámico del músculo. En cuanto a la fuerza muscular hubo una mejoría en el puntaje de los extensores de tronco, pasando de hacer el movimiento a favor de la gravedad a realizarlo parcialmente contra la gravedad según escala MRC. Igualmente, en los dorsiflexores y plantiflexores de tobillo bilateralmente, se mejoró el puntaje, siendo el resultado del último grupo muscular mencionado el más significativo, ya que evolucionó de mostrar contracción muscular visible sin movimiento, a realizarlo a favor de la gravedad. Los demás grupos musculares evaluados mantuvieron el puntaje inicial.

Por otra parte, la flexibilidad fue evaluada por medio de diferentes pruebas semiológicas y la medición se apoyó de la medición de goniometría para identificar los grados de deficiencia en la movilidad, producto de la disminución de la flexibilidad. En general la paciente presentó disminución en el grado de retracción y ganancia de amplitud del movimiento en los distintos grupos musculares; se evidenció una mejoría significante en la flexibilidad de los flexores de cadera bilateralmente, y a nivel de los flexores de rodilla del lado derecho, evidenciando ganancia de rango de movilidad y pasando de una retracción moderada a ligera.

En cuanto al desempeño de la función motora gruesa, se mantuvo la clasificación del nivel III de desempeño en la GMFM-CS, ya que posterior a la intervención continúa requiriendo caminador posterior fuera de casa y en algunas ocasiones dentro de ella para sus desplazamientos. Por el contrario, en el desempeño del equilibrio estático y dinámico presentó cambios, los cuales se vieron reflejados en los resultados de las siguientes pruebas: en la prueba de alcance funcional, inicialmente la paciente realizó $5 \mathrm{~cm}$ de desplazamiento anterior, y posterior a la intervención alcanzó un desplazamiento anterior de $7 \mathrm{~cm}$, evidenciándose una ganancia de $2 \mathrm{~cm}$, lo cual indica una disminución de riesgo de caída por pérdida de equilibrio.

A esto se le añade el resultado obtenido en el test de levántate y anda (TUG), para el cual obtuvo en la evaluación pre-intervención un tiempo total de 25 segundos, reduciéndose en la evaluación post-intervención, mejorándolo en 4 segundos, 
diferencia que se obtuvo por un mejor desempeño en el momento de la transferencia de la silla al bípedo con apoyo en el caminador posterior, mostrando mayor seguridad y rapidez durante este movimiento. En cuanto al test de los 6 minutos en la evaluación previa a la intervención, realizó 154 metros, recorrido que aumentó 38 metros en la evaluación final, para un total de 192 metros, indicando cambios significativos en la distancia recorrida.

En la escala de balance pediátrica de Berg, presentó una mejoría en 9 de las categorías evaluadas, se destaca su mejor desempeño en la transferencia de bípedo a sedente y viceversa, en esta fue capaz de levantarse y sentarse de forma independiente usando sus manos, en el mantenimiento de la posición bípeda con ojos cerrados, donde fue capaz de mantener la posición durante 54 segundos con seguridad, en el mantenimiento del bípedo con pies juntos, el cual realizó bajo supervisión manteniéndolo por 32 segundos, y en la inclinación anterior con pies apoyados. En esta pudo inclinarse hacia adelante menos de $12,5 \mathrm{~cm}$ y mantuvo el equilibrio. Las otras categorías mantuvieron el puntaje inicial. Por tal motivo el puntaje total postintervención superó en 12 puntos a la inicial, lo cual indicó una mejoría tanto en el equilibrio estático como dinámico.

\section{DISCUSIÓN}

En el test de caminata de los 6 minutos, se encontró una ganancia en la distancia de 38 metros, lo cual revela una mejoría en la capacidad funcional de la paciente en cuanto a velocidad y distancia de desplazamiento, significando una mayor capacidad para realizar sus actividades de la vida diaria con un menor gasto energético y a su vez una mayor tolerancia al esfuerzo, lo que se traduce en un impacto positivo sobre su calidad de vida (11).

Los niños con parálisis cerebral que tienen compromiso del tono muscular y deficiencia en el control postural, presentan riesgo de caídas, esto es influenciado por la deficiente coordinación entre los grupos musculares, uno de ellos los anti gravitatorios, la deficiencia en las sinergias musculares y el pobre desempeño de las reacciones posturales (12). La paciente del caso presentó una ganancia de $2 \mathrm{~cm}$ en la prueba de alcance anterior, lo cual revela una mayor estabilización de sus miembros inferiores y pelvis, mayor coactivación a nivel de la musculatura antigravitatoria de miembros inferiores y tronco, y un mejor desempeño de las reacciones de equilibrio y enderezamiento, permitiéndole mayor control del movimiento durante la inclinación del cuerpo en sentido anterior, lo cual contrarresta el riesgo de caída.

Según la versión de Franjoine et al, un paciente que presente moderado riesgo de caídas, presentará mal adaptaciones que tienen como efectos secundarios restricción de movilidad, lo que a su vez influye a que siga decreciendo el equilibrio y además a una restricción en la participación, por lo tanto, puede haber un declive funcional y una disminución de la calidad de vida, ya que muchos estudios han demostrado que los síntomas motores contribuyen al empeoramiento de la percepción de la misma (13) (14). 
Actualmente, las intervenciones o terapias más estudiadas en los últimos años que tienen el objetivo de mejorar el control postural y equilibrio en niños con PC son muy variadas, entre ellas: la realidad virtual (RV), hipoterapia o actividades asistidas con caballos y el entrenamiento en caminadora (15). Grecco et al. realizó un ECA con 15 niños con PCI de 3-12 años, su objetivo fue comparar los efectos del entrenamiento del equilibrio con trabajo dinámico sobre caminadora con el entrenamiento en el suelo sobre el equilibrio funcional y estático de los pacientes, con un sistema de posturografía y la escala de Berg. Ambos protocolos de entrenamiento de la marcha condujeron a una mejora dentro del grupo en el equilibrio funcional. Sin embargo, el entrenamiento en la caminadora condujo a un aumento medio de 14.7 puntos en la escala de equilibrio de Berg, mientras que el entrenamiento en tierra condujo a un aumento de 3.2 puntos (16).

En contraste con estos resultados, en el presente estudio se encontró una mejora de 12 puntos en la escala de equilibrio pediátrica de Berg, al comparar los resultados pre y post intervención, lo cual deja en evidencia la eficacia del estabilizador distal o Mini standing, sobre la mejora del equilibrio estático y dinámico, siendo una estrategia de mayor accesibilidad y bajo costo, comparada con el entrenamiento en caminadora.

Por último, con relación a los resultados obtenidos en el paciente en cuanto a su fuerza muscular, aunque se destaca el mejor desempeño de los extensores de tronco, no se observaron cambios llamativos de manera global. Es claro que para lograr beneficios positivos sobre la funcionalidad se hace necesario asegurar una serie de adaptaciones morfológicas y fisiológicas del sistema neuromuscular que faciliten un incremento de la masa muscular (17).

\section{CONCLUSIONES}

La Parálisis cerebral es la primera causa de discapacidad en la infancia y genera importantes repercusiones a nivel social, económico, sanitario, educativo y familiar. El control postural de los niños con PC está disminuido, lo que ocasiona grandes consecuencias en la bipedestación y la marcha. De acuerdo con los resultados obtenidos en el presente estudio, se logró demostrar la eficacia que tuvo el uso del estabilizador distal o Mini standing en un caso de una niña con PC diparesia espástica, como estrategia para favorecer un mejor desempeño de las reacciones posturales, la coordinación muscular, el equilibrio y control postural, generando beneficios en el funcionamiento tanto en la parte motora, como psicosocial de la paciente.

Se pudo evidenciar una progresión en las diferentes variables evaluadas, por lo cual se debe considerar este método tipo ortesis como una buena estrategia, económica, accesible y eficaz para implementar en casa con ayuda de un profesional en fisioterapia y de su familia o cuidador, como elemento facilitador de los procesos terapéuticos, con el objetivo de aumentar la funcionalidad y por ende la autoestima del niño, mejorando su participación en la sociedad y su calidad de vida. 
1. C, Calzada. C V. Parálisis cerebral infantil: definición y clasificación a través de la historia. Rev Mex Ortop PEDIÁTRICA [Internet]. 2014;16(1):6-10. Available from: http://www.medigraphic.com/opediatria

2. Cano de la Cuerda R. Martínez Piédrola R. Miangolarra Page J. Control y Aprendizaje Motor. 2016. p. 1-6.

3. Delgado María DA. Síndrome de Cockayne. 2015.

4. Macías Merlo ML. Fisioterapia en Pediatria. 2002. p. 461.

5. M. José Fernández. Descubriendo La Parálisis Cerebral. 2000;68. Available from: www.aspace.org

6. Bartlett D, Birmingham T. Validity and reliability of a pediatricreach test. Pediatr Phys Ther. 2003; 15:84---92.

7. N Gómez-Regueira. Viñas S. Mejora del control postural y equilibrio en la parálisis cerebral infantil: revisión sistemática. ELSEVIER. 2016;38(4)

8. Nogueira TC, Carbonero FC, da Silva FP, Silva KR. Uso doNintendo®Wii para reabilitac,ão de crianc, as com paralisia cere-bral: estudo de caso. Rev Neurocienc. 2013; 21:286----9

9. Palisano R, Rosenbaum P, Walter S, Russell D, Wood E, Galuppi B. Development and reliability of a system to classify gross motor function in children with cerebral palsy. Dev Med Child Neurol. 1997; 39:214-23

10. Flórez MR, Ostrosky-Solís F. Atención y Memoria en Pacientes con Parálisis Cerebral Infantil. Rev Neuropsicol Neuropsiquiatría y Neurociencias. 2009;9(1):55-64.

11. Barona, O. Díaz, G. Caminata de seis minutos: propuesta de estandarización del protocolo y aplicación práctica para la evaluación de la hipertensión pulmonar con especial referencia a la de los niños. ELSEVIER. 2016; 23(1): 59-67.

12. Soares, L. Savazzi, J. Carvalho, R. Rendimiento motor de niños con parálisis cerebral en alcance anterior. ELSEVIER.2019;68: 158-162. Disponible en: https://www.clinbiomech.com/article/S0268-0033(19)30034-8/fulltext

13. Serrano, M. Forero, J. Efectos de la terapia física intensiva sobre la función motora de un niño con hemiparesia espástica. Rev. Fac. Med. 2016 Vol. 64 Supl:57-63.

14. Quiñones, K. Robayo, A. García, A. Diseño de un prototipo de bipedestador para pacientes pediátricos con espina bífida. Rev. Fac. Med. 2013 Vol. 61 No. 4: 423-429.

15. Gómez, N. Viñas, S. Mejora del control postural y equilibrio en la paralisis cerebral infantil: revision sistematica. ELSEVIER.2016;38(4):196-214.

16. Grecco, L. Tomita, S. Christovao, T. Efecto del entrenamiento de la marcha en cinta rodante sobre el equilibrio estático y funcional en niños con paraáisis cerebral: un ensayo controlado aleatorio. Rev. Brasileña de Fisioterapia. 2013;17:17-23.

17. Ocampo, N. Ramírez, J. El efecto de los programas de fuerza muscular sobre la capacidad funcional. Rev. Fac. Med. 2018 Vol. 66 No. 3: 399-410. 